\title{
Indicadores de eficiência técnica e econômica do milho cultivado em sistema plantio direto no Estado de Santa Catarina, Brasil ${ }^{1}$
}

\author{
Flávio José Simioni ${ }^{*}$, Marie Luise Carolina Bartz, Leandro do Prado Wildner ${ }^{4}$, Evandro Spagnollo ${ }^{4}$, \\ Milton da Veiga ${ }^{5}$, Dilmar Baretta
}

10.1590/0034-737X201764030003

\begin{abstract}
RESUMO
Este trabalho teve como objetivo avaliar os indicadores de eficiência técnica e econômica da produção de milho, em três variações de manejo em sistema plantio direto (lavoura com apenas adubação mineral - PDM; lavoura com adubação mineral e orgânica - PDO e integração lavoura e pecuária - PDI) e suas relações com os atributos físicos e químicos do solo. O estudo foi realizado em lavouras de sete municípios do Estado de Santa Catarina, determinandose os custos de produção e os indicadores técnicos e econômicos das culturas. Em cada lavoura selecionada, foram coletadas, em três pontos equidistantes, amostras para avaliações dos atributos químicos e físicos do solo na camada de $0-10 \mathrm{~cm}$. Os dados foram submetidos a análises univariada e multivariada. Os resultados indicaram que o sistema PDM apresenta os maiores custos de produção, enquanto o PDO apresenta os melhores resultados econômicos e de eficiência de produção. Não foram observados efeitos significativos dos diferentes manejos, em sistema plantio direto, sobre os atributos químicos e físicos do solo. Contudo, a análise multivariada possibilitou mostrar, de modo mais amplo, as relações entre as variáveis estudadas e os diferentes manejos, em sistema plantio direto.
\end{abstract}

Palavras-chave: custos de produção; adubação orgânica; integração lavoura-pecuária.

\section{ABSTRACT}

\section{Technical and economic efficiency indicators of corn grown in no-tillage system in the State of Santa Catarina, Brazil}

This study aimed to evaluate the technical and economic efficiency indicators of the production of corn in three management variations in no-tillage system (tillage, with mineral only fertilization - PDM; tillage, with mineral and organic fertilizing - PDO; and integrated crop-livestock - PDI) and their relationship with physical and chemical attributes of the soil. The study was conducted in corn crops located in seven cities in the state of Santa Catarina, determining the cost of production and the technical and economic indicators of culture. In each selected crop, composite samples were collected, in three equidistant points, for the evaluation of chemical and physical attributes of the soil at a depth of $0-10 \mathrm{~cm}$. The data were subjected to univariate and multivariate analyses. The results indicate that the PDM system presents the highest production costs, while the PDO offers the best economic results and productive efficiency. No significant effects were observed in the different managements in no-tillage systems on soil chemical and physical attributes. However, multivariate analysis made possible to show, more broadly, relations among the variables studied and different management in no-tillage systems.

Key words: integrated crop-livestock; organic fertilizing; production costs.

\footnotetext{
Submetido em 10/12/2014 e aprovado em 16/05/2017.

Este trabalho é parte dos resultados da pesquisa financiada pela Fundação Agrisus.

${ }^{2}$ Universidade do Estado de Santa Catarina, Programa de Pós-Graduação em Ciências Ambientais, Lages, Santa Catarina, Brasil. flavio.simioni@udesc.br

3 Universidade Positivo, Programa de Pós-Graduação em Gestão Ambiental, Curitiba, Paraná, Brasil. bartzmarie@gmail.com

${ }^{4}$ Empresa de Pesquisa Agropecuária e Extensão Rural de Santa Catarina, Centro de Pesquisa para Agricultura Familiar, Chapecó, Santa Catarina, Brasil. lpwild@epagri.sc.gov.br; spagnollo@epagri.sc.gov.br

${ }^{5}$ Empresa de Pesquisa Agropecuária e Extensão Rural de Santa Catarina, Estação Experimental de Campos Novos, Campos Novos, Santa Catarina, Brasil. milton.veiga.br@gmail.com

${ }^{6}$ Universidade do Estado de Santa Catarina, Centro de Educação Superior do Oeste, Departamento de Zootecnia, Chapecó, Santa Catarina, Brasil. dilmar.baretta@udesc.br

*Autor para correspondência: flavio.simioni@udesc.br
}

Rev. Ceres, Viçosa, v. 64, n.3, p. 232-241, mai/jun, 2017 


\section{INTRODUÇÃO}

A cultura do milho (Zea mays) tem grande importância socioeconômica, sendo a produção de grãos destinada à fabricação de rações para a alimentação animal, além de atender a necessidade de consumo na dieta diária de uma parcela significativa da população. A produção de milho, nos cenários brasileiro e catarinense, apresenta tendências bem distintas. Enquanto no Brasil, observa-se um aumento da produção da cultura, elevando o país ao posto de terceiro maior produtor mundial (MAPA, 2014), Santa Catarina apresenta redução da sua área plantada nos últimos anos, passando de 816,1 para 484,4 mil ha, entre as safras de 2003-04 e de 2012-13 (IBGE, 2014).

A relação desfavorável entre preço do milho e o da soja (Glicine max L.) é um dos principais fatores que explica a queda da área cultivada com milho, em Santa Catarina. Numa perspectiva de longo prazo, a perda da atratividade econômica da produção de milho para os pequenos agricultores, as mudanças no sistema de produção de suínos e a substituição das áreas de milho-grão, para a produção de alimentos, por áreas de produção leiteira (pastagens e silagem) explicam a redução da área de milho (EPAGRI, 2014a). Apesar do significativo crescimento da produtividade no mesmo período (cerca de $70 \%$ em 9 anos), em grande parte graças à seleção das melhores áreas para seu cultivo, a produção não é suficiente para atender a demanda destinada à produção animal, aumentando o déficit de milho em Santa Catarina.

Neste cenário, é importante considerar sistemas que apresentem maior eficiência de produção para aumentar a competitividade da cultura e proporcionar maior rentabilidade ao produtor. Vários trabalhos mostram a relação entre a eficiência produtiva da agricultura brasileira e a incorporação de tecnologias, como relatam Cusumano et al. (1975) e Silva et al. (1983), ao avaliarem os efeitos da mecanização, e Kageyama (2003) e Silveira et al. (2001), ao correlacionarem a adoção de tecnologia e o aumento da produtividade. De maneira mais ampla, Conterato et al. (2012) verificaram que a eficiência produtiva da agropecuária brasileira aumentou 3,7 vezes no período de 1975 a 2010, sobretudo após 1995.

Considerando-se os dados de custo de produção para a cultura do milho apresentados pelo Centro de Socioeconomia e Planejamento Agrícola (EPAGRI, 2014b), a produção de alta tecnologia apresenta redução de 5,25\% do custo operacional direto, em comparação com a produção de média tecnologia. No Estado do Paraná, o sistema plantio direto (SPD) proporciona custo total médio 17,3\% menor que o do sistema de produção convencional (SEAB, 2013).

Considerando-se a eficiência técnica, o SPD proporcionou produtividade da cultura do milho (Cruz et al.,
2009; Kaneko et al., 2010) e do milho safrinha (Possamai et al., 2001), maior que a de outros sistemas de preparo do solo.

No que tange à rentabilidade, o SPD proporcionou lucratividade para rotação de culturas (Santos et al., 2004), maior do que a proporcionada pelo preparo convencional do solo, para a cultura do milho (Kaneko et al., 2010) e para a cultura da soja (Alvim et al., 2004).

O uso do SPD resulta em um agroecossistema com grau de perturbação menor que o de outras formas de manejo que usam intensa mobilização do solo. Isso se justifica pelo fato de que este complexo de tecnologias exige menos trabalho e menos energia fóssil, estimula os processos de agregação do solo, reduz as taxas de mineralização da matéria orgânica, reduz a erosão e promove o controle biológico de pragas, doenças e plantas daninhas, diminuindo a utilização de agrotóxicos. Desta forma, há um significativo estímulo para a recomposição da biodiversidade do solo, como a do ambiente em si, resultante do menor impacto humano no sistema (Derpsch et al., 1991; Pieri et al., 2002; Brown et al., 2003; Baretta et al., 2003; Bartz et al., 2013; Bartz et al., 2014).

Diante do exposto, o objetivo deste trabalho foi avaliar os indicadores de eficiência técnica e econômica da produção de milho, em três variações de manejos em SPD (lavoura com apenas adubação mineral - PDM; lavoura com adubação mineral e orgânica - PDO e integração lavoura-pecuária - PDI), bem como estabelecer as relações entre estes indicadores e os atributos físicos e químicos do solo.

\section{MATERIAL E MÉTODOS}

\section{Região de estudo}

A área de abrangência do estudo constou de amostragens em dez propriedades rurais, representativas de sistemas de cultivo de culturas anuais, no Estado de Santa Catarina, localizadas nos municípios de Guaraciaba (1), Maravilha (2), Guarujá do Sul (1), Chapecó (2), Pinhalzinho (1), Guatambu (1) e Campos Novos (2). As lavouras amostradas foram consideradas réplicas verdadeiras dos sistemas estudados, conforme recomenda Pereira et al. (2013), porque foi garantido que os mesmos tratamentos (sistemas e áreas) fossem repetidos nos municípios e regiões estudadas.

\section{Manejo do solo}

Para este estudo, foram selecionadas lavouras com mais de cinco anos de uso continuado do SPD, visando a atender aos princípios de qualidade desse sistema, amostrando-se três modalidades de manejo, em SPD, representativas da região Oeste de Santa Catarina: lavoura 
com o uso apenas de adubação mineral (PDM, $\mathrm{n}=4$ ), lavoura com uso de adubação mineral e orgânica (PDO, n $=3$ ) e integração lavoura-pecuária (PDI, $n=3$ ). Os sistemas PDM e PDO caracterizam-se pelo cultivo de grãos na safra e entressafra, utilizando apenas adubação mineral para a fertilização (PDM) ou adubação mineral mais orgânica (cama de aviário ou dejeto líquido de suínos), sendo a adubação orgânica aplicada usualmente antes da semeadura das culturas de inverno e de verão. Já o sistema PDI caracteriza-se pelo cultivo de grãos na safra e pastagens de inverno (aveia e azevém) na entressafra, esta utilizada para alimentação de bovinos em sistema de pastejo rotativo.

\section{Variáveis: atributos químicos e físicos do solo}

Em cada lavoura selecionada, foram coletadas, em três pontos equidistantes, amostras compostas para a determinação dos atributos químicos do solo, na camada de 0 a $10 \mathrm{~cm}$. Os atributos químicos avaliados foram; pH em água; teor de matéria orgânica (MO); fósforo extraível $(\mathrm{P})$; potássio $(\mathrm{K})$; alumínio $(\mathrm{Al})$; cálcio $(\mathrm{Ca})$ e magnésio $(\mathrm{Mg})$ trocáveis, conforme metodologia descrita em Tedesco et al. (1995). Os atributos físicos foram determinados em amostras coletadas com estrutura preservada em anéis volumétricos, na camada de 2 a $7 \mathrm{~cm}$, nos mesmos pontos das coletas para análises químicas, determinando-se: densidade do solo (DS); resistência à penetração (RP); volume total de poros (PT); volume de macroporos (MacroP) - poros com diâmetro maior do que 50 micrômetros; volume de microporos (MicroP) poros com diâmetro menor que 0,5 micrômetros; diâmetro médio geométrico dos agregados secados ao ar (DMG), utilizando-se metodologias de rotina descritas em Veiga (2011).

\section{Determinação dos custos de produção}

Os indicadores econômicos foram obtidos para a cultura do milho-safra (cultura principal) e o cálculo dos itens que compõem o custo de produção foi elaborado com base nas metodologias utilizadas, em Santa Catarina, pela EPAGRI (2014b); no Paraná, pela SEAB (2013); no Brasil, pela Companhia Nacional de Abastecimento (CONAB, 2010) e nos conceitos teóricos apontados por Hoffmann et al. (1981). A coleta de dados técnicos e econômicos foi realizada por meio de visitas com entrevistas aos produtores e pela identificação dos componentes de custo, em planilha eletrônica, considerando-se o milho cultivado na safra do ano agrícola 2012-2013 e tendo, como referência, os preços praticados em Santa Catarina, no mês de maio de 2013.

Na determinação dos custos variáveis (CV), foram considerados: a) Insumos - sementes, adubos e defensivos, valorados a preço de mercado regional, considerando-se os do mês de maio de 2013 como referência;

b) Serviços com mão de obra - execução de todos os serviços que demandam mão de obra, como semeadura, pulverizações, adubações e colheita, tendo como base o tempo das operações informado pelos agricultores (o valor da hora-homem foi determinado considerando-se o custo de oportunidade do trabalho no mercado, calculado a partir de uma remuneração de dois salários mínimos mensais, totalizando R \$1.356,00, mais encargos de $45 \%$, com jornada de trabalho de 176 horas/mês, o que resultou em 11,17 R \$/hora);

c) Serviços mecânicos - considerou-se a execução de todos os serviços que demandam horas-máquina, mais implementos, para a execução das operações de semeadura, pulverizações, adubações e colheita. Considerouse o custo variável de mercado da hora-máquina, mais implementos, para todas as operações realizadas, exceto o valor correspondente ao operador, uma vez que este foi incluído nos serviços com mão de obra, a partir das estimativas realizadas pelo CEPA/EPAGRI, tendo como base o tempo das operações informado pelos agricultores. O tempo relativo ao transporte interno de produtos utilizados em cada operação também foi incluído; para a operação de semeadura foi considerada a utilização de um trator de $120 \mathrm{CV}$, enquanto para as demais operações utilizou-se o custo relativo a um trator de $75 \mathrm{CV}$; para a operação de colheita considerou-se uma colhedora média;

d) Despesas gerais - valor estimado, considerando-se 1,0\% de insumos, serviços com mão de obra e serviços mecânicos;

e) Assistência técnica - valor estimado, considerando-se 2,0\% de insumos, serviços de mão de obra, serviços mecânicos e despesas gerais;

f) Seguro da produção - PROAGRO - valor estimado, considerando-se $3,9 \%$ de insumos, serviços com mão de obra, serviços mecânicos e despesas gerais;

g) Custos financeiros (Cf) - valor estimado, considerando-se taxa de juros de 0,5\% ao mês sobre o valor Parcial dos custos variáveis - PCV (insumos, serviços de mão de obra, serviços mecânicos, despesas gerais, assistência técnica e seguro da produção), capitalizados durante sete meses pela expressão: $\mathrm{Cf}=\left[\mathrm{PCV}(1,005)^{7}\right]$ PCV;

h) Despesas com a comercialização - para o transporte externo, foi considerado o valor médio informado pelos agricultores ( $\mathrm{R} \$$ /saca) e para o item previdência social (Funrural), foram aplicados 2,3\% sobre a receita bruta. 
Na determinação dos custos fixos (CF), foram considerados:

a) Manutenção de benfeitorias - foi considerado como benfeitoria um galpão rústico utilizado para máquinas e equipamentos e para armazenagem dos insumos, tomando-se como base a área construída, em $\mathrm{m}^{2}$, informada pelo agricultor e o valor médio de 200,00 R \$/ $\mathrm{m}^{2}$ (o cálculo da manutenção considerou $1,0 \%$ do valor novo (VN), dividido pela área cultivada com lavouras da propriedade e rateado para duas culturas anuais);

b) Depreciação de benfeitorias - obtida pelo método linear, com base nos valores de VN, valor residual (VR) e vida útil (VU), dada por $\mathrm{D}=(\mathrm{VN}-\mathrm{VR}) / \mathrm{VU} / a ́$ rea cultivada, rateados para duas culturas anuais. Como VN foi considerado o valor de $200,00 \mathrm{R} \$ / \mathrm{m}^{2}$, como VR o valor de $20 \%$ do VN e como VU o tempo de 25 anos;

c) Depreciação de máquinas e equipamentos - obtida pelo método linear por $\mathrm{D}=(\mathrm{VN}$ - VR)/VU. Como VN foi considerado o valor atual de mercado, VR de $10 \%$ ou $20 \%$ do VN, conforme o equipamento considerado e, VU conforme indicação do fabricante;

d) Seguro de máquinas - a taxa de seguro foi de $1 \%$ ao ano, considerando-se o uso médio anual, em horas/ano, obtido por $\mathrm{S}=(\mathrm{VN}+\mathrm{VR}) / 2 * 0,01 / \mathrm{Uso}$ anual;

e) Correção do solo - obtida considerando-se o valor do calcário, acrescido do custo de distribuição (horas-homem mais horas-máquina: trator mais implemento), dividido pelo intervalo de aplicação em anos, rateado entre duas culturas anuais;

f) Impostos e taxas - valor estimado, considerando-se $0,05 \%$ sobre o valor da terra, rateado para duas culturas anuais;

g) Remuneração do capital máquinas e equipamentos - representa o custo de oportunidade da hora-máquina, obtido a partir da aplicação da taxa de $6 \%$ ao ano sobre o valor médio, considerando-se o uso médio anual, em horas/ano, obtido por $\mathrm{RC}=(\mathrm{VN}+\mathrm{VR}) / 2 * 0,06 / \mathrm{Uso}$ anual;

h) Remuneração do capital benfeitorias - representa o custo de oportunidade do galpão, obtido a partir da aplicação da taxa de $6 \%$ ao ano sobre o valor médio [(VN+VR)/2] e rateado pela área cultivada e por duas culturas anuais;

i) Remuneração do empresário (RE) - representa o custo de oportunidade do empreendedor (proprietário), obtido considerando-se $8 \%$ sobre o Custo Variável Total;

j) Remuneração da terra (RT) - representa o custo de oportunidade do capital terra, obtido considerando-se $1 \%$ sobre o valor da terra, rateado para duas culturas anuais.
O custo operacional total (COT) foi obtido considerando-se os custos variáveis totais (CVT) e parte dos custos fixos (itens a até f). O custo total (CT) é a soma dos custos variáveis e fixos totais $(\mathrm{CT}=\mathrm{CV}+\mathrm{CF})$.

\section{Determinação dos indicadores de eficiência técnica e econômica}

O cálculo dos indicadores considerou a utilização do solo com duas culturas anuais, sendo uma na entressafra (inverno) e outra na safra (verão). Os indicadores de eficiência econômica no curto prazo foram:

- Receita bruta (RB), que é a quantidade produzida (q), valorada pelo preço de mercado praticado (p) em maio de $2013(\mathrm{RB}=\mathrm{q} * \mathrm{p})$;

- Margem bruta (MB = RB - CV), o saldo (valor) destinado à remuneração dos custos fixos;

- Renda líquida ( $\mathrm{RL}=\mathrm{RB}-\mathrm{COT})$, o saldo (valor) destinado à remuneração dos custos de oportunidade (do capital e do empreendedor);

- Lucro $(\mathrm{L}=\mathrm{RB}-\mathrm{CT})$, o resultado final após a remuneração de todos os fatores de produção;

Ponto de nivelamento $(\mathrm{PN}=\mathrm{CT} /$ preço $)$, a quantidade de produção necessária para cobrir o custo total;

Taxa de remuneração da terra $(\mathrm{TRT}=\mathrm{L}+\mathrm{RT} / \mathrm{valor}$ da terra), que representa a remuneração que o fator de produção terra obteve após a compensação de todos os demais fatores de produção; e

- Produtividade total dos fatores $(\mathrm{PTF}=\mathrm{RT} / \mathrm{CT})$, uma medida de eficiência, isto é, indica o número de unidades monetárias recebidas para cada unidade monetária de custo.

Os indicadores de eficiência técnica foram:

- Produtividade por área (PRODA), medida em kg/ha;

Produtividade da mecanização (PRODMEC), obtida pela divisão da PRODA pelo total de horas-máquina utilizadas no cultivo do milho, medida em kg/ha/hora-máquina;

Produtividade da fertilização, obtida pela divisão da PRODA pelo total de nitrogênio (PROD-N, kg/ha/kg de $\mathrm{N}$ ), de fósforo (PROD-P, $\mathrm{kg} / \mathrm{ha} / \mathrm{kg}$ de $\mathrm{P}_{2} \mathrm{O}_{5}$ ) e de potássio (PROD-K, kg/ha/kg de $\mathrm{K}_{2} \mathrm{O}$ ).

\section{Tratamento estatístico}

Inicialmente, todas as variáveis dos três tipos de manejo em SPD (PDM, PDO e PDI) foram submetidas à análise de variância, considerando-se o delineamento inteiramente casualizado e comparação de médias pelo teste de Student-Newman-Keuls (SNK). Posteriormente, os dados foram submetidos a métodos de análise estatística 
multivariada, considerando-se os indicadores técnicos e econômicos (CVT, CFT, CF-A, CF-B, COT, CT, RB, MB, RL, LUCRO, PN, TRT, PTF, PRODA, PRODMEC, PRODN, PROD-P E PROD-K) como variáveis respostas e os atributos químicos (pH, P, K, MO, Al, Ca e Mg) e físicos do solo (DS, RP, PT, MacroP, MicroP e DMG) como variáveis explicativas.

A amplitude do gradiente dos dados foi menor do que três $(0,280)$, indicando que cada variável assume uma resposta linear em relação ao eixo (gradiente) (Leps \& Smilauer, 1999). Neste caso, de acordo com os mesmos autores, recomenda-se o uso da Análise de Componentes Principais (ACP), de modo a distinguir (separar) os tratamentos (PDM, PDO e PDI) a partir dos seus componentes principais. Os procedimentos de análise foram realizados conforme Braak \& Smilauer (1998). As variáveis explicativas foram adicionadas, a posteriori, para relacioná-las com as variáveis respostas, visualizando-se assim a estrutura subjacente dos dados, ou seja, a relação entre os atributos físicos e químicos do solo com os indicadores de eficiências técnica e econômica da cultura do milho.

\section{RESULTADOS E DISCUSSÃO}

Considerando-se os componentes do custo de produção para a cultura do milho (Tabela 1), foram observadas diferenças significativas apenas para o custo operacional total (COT), com menor valor no PDI e maior no PDM. Em relação ao sistema PDM, o PDO e o PDI apresentaram reduções de 8,0 e de $12,1 \%$ no COT, respectivamente. Os principais fatores que explicam as diferenças observadas foram as diferenças na adubação mineral na base (de $400 \mathrm{~kg} / \mathrm{ha}$ de NPK, no PDM, para $350 \mathrm{~kg} / \mathrm{ha}$, no PDI, e $275 \mathrm{~kg} / \mathrm{ha}$, no PDO) e em cobertura (de $462 \mathrm{~kg}$ / ha, no PDM, para $350 \mathrm{~kg} / \mathrm{ha}$, no PDI e, no PDO), apesar do sistema PDO apresentar custo adicional com máquinas para a distribuição do adubo orgânico.

Os resultados de custo de produção obtidos neste trabalho assemelham-se aos custos de produção do milho cultivado em SPD, calculados pela Secretaria da Agricultura do Estado do Paraná, para fevereiro de 2013 (SEAB, 2013), de 2.867,80 R \$/ha, considerando-se uma produtividade esperada de $7.200 \mathrm{~kg} / \mathrm{ha}$. Por outro ladro, o custo dos insumos foi inferior ao calculado pelo CEPA/EPAGRI, para produção de milho, em Santa Catarina, considerando-se plantio direto com semente transgênica e produtividade esperada de $9.900 \mathrm{~kg} / \mathrm{ha}$, que foi $\mathrm{R} \$ 1.937,82$, em agosto de 2013 (EPAGRI, 2014b).

Não houve diferenças significativas entre os sistemas de manejo do solo sob plantio direto, para a maioria dos indicadores técnicos e econômicos (Tabela 2), diferentemente dos resultados obtidos por outros autores. Gomes et al. (2005) verificaram que a adubação orgânica promo- ve incrementos dos componentes de produção de milho e que uma dose de $40 \mathrm{~m}^{3} / \mathrm{ha}$ apresentou produção semelhante à dose de $500 \mathrm{~kg} / \mathrm{ha}$ do adubo NPK. Diferenças significativas foram observadas na resposta do $\mathrm{N}$ e do $\mathrm{P}$, quando se comparou o sistema PDO com os demais tratamentos, o que pode ser explicado pela dinâmica de liberação dos nutrientes de fonte orgânica, que ocorre num intervalo de tempo maior do que para o adubo solúvel, aumentando a eficiência de absorção (SBCS, 2016).

Também não foram observadas diferenças significativas entre as modalidades de plantio direto, para os atributos químicos e físicos do solo estudados, com exceção dos teores de P (Tabela 3). Para os atributos químicos, a ausência de diferenças pode ser explicada pelo alto coeficiente de variação. No caso dos atributos físicos, por sua vez, esse resultado pode ser explicado pela época de coleta das amostras, definida em função de se diagnosticar o efeito de longo prazo do manejo do solo, pois, segundo Veiga et al. (2014), a compactação promovida pelo pisoteio de bovinos concentra-se na camada superficial do solo $(<10 \mathrm{~cm})$ e se desfaz durante o ciclo da cultura subsequente à pastagem, em função da decomposição do sistema radicular da pastagem e da ocorrência de ciclos de umedecimento e secagem do solo.

A análise estatística convencional não foi eficiente para diagnosticar diferenças entre as modalidades do sistema plantio direto estudadas, principalmente, em função do alto coeficiente de variação entre as lavouras amostradas. Em razão disso, foi utilizada a análise multivariada, que viabiliza a análise conjunta dos dados e os setoriza em quatro quadrantes, possibilitando a visualização e a mensuração das diferenças entre as modalidades.

A análise multivariada, para as variáveis respostas (indicadores técnicos e econômicos), mostrou que os eixos um e dois da PCA explicaram, respectivamente, 54,0 e 26,7\% da variabilidade dos dados, totalizando $80,7 \%$ de sua variabilidade total (Figura $1 \mathrm{~A}$ ). Considerando-se somente as variáveis respostas selecionadas (PN, CVT, PRODA, LUCRO, PRODN, PTF), os eixos um e dois da PCA explicaram, respectivamente, 68,8 e $25,7 \%$ da variabilidade dos dados, totalizando $94,6 \%$ da variabilidade total dos dados (Figura 1 B). Os valores médios dos componentes principais, representados por meio dos centroides das modalidades de plantio direto (PDM, PDO e PDI), indica a nítida separação entre os três tratamentos avaliados.

De acordo com a Figura 1, o plantio direto com adubação orgânica (PDO) está associado ao melhor resultado econômico da cultura do milho (RB, MB, RL, LUCRO), à maior eficiência em termos de produtividade (PRODA, PROD-P e PROD-N) e à melhor remuneração dos fatores empregados (PTF) e da terra (TRT). Por outro lado, o PDM está associado ao maior custo de produção (CVT, COT, 
Tabela 1: Custo de produção do milho em área de plantio direto com adubação mineral e orgânica (PDO), com apenas adubação mineral (PDM) e com Integração Lavoura-Pecuária (PDI)

\begin{tabular}{|c|c|c|c|}
\hline \multirow[b]{2}{*}{ Especificação (R\$/ha) } & \multicolumn{3}{|c|}{ Sistema plantio direto } \\
\hline & $\begin{array}{c}\text { PDO } \\
\text { (com adubação mineral } \\
\text { e orgânica) }\end{array}$ & $\begin{array}{c}\text { PDM } \\
\text { (com apenas adubação } \\
\text { mineral) }\end{array}$ & $\begin{array}{c}\text { PDI } \\
\text { (Integração Lavoura- } \\
\text { Pecuária) }\end{array}$ \\
\hline Custo variável total (CVT) & $2.259,42 a$ & $2.524,59 a$ & $2.182,91 a$ \\
\hline 1 - Insumos & $1.439,48$ & $1.812,34$ & $1.545,54$ \\
\hline 2 - Serviços de mão de obra & 55,33 & 37,42 & 38,46 \\
\hline 3 - Serviços mecânicos & 255,17 & 227,74 & 172,19 \\
\hline 4 - Despesas gerais & 17,50 & 20,77 & 17,56 \\
\hline 5 - Assistência técnica & 35,35 & 41,97 & 35,48 \\
\hline 6 - Seguro da produção (Proagro) & 68,93 & 81,83 & 69,18 \\
\hline 7 - Custos financeiros & 66,50 & 78,95 & 66,74 \\
\hline 8 - Despesas de comercialização & 321,16 & 223,58 & 237,76 \\
\hline Custo fixo total (CFT) & $596,90 a$ & $601,60 a$ & $534,69 a$ \\
\hline 1 - Manutenção de benfeitorias & 3,67 & 2,38 & 1,01 \\
\hline 2 - Depreciação de benfeitorias & 11,75 & 7,63 & 3,23 \\
\hline 3 - Depreciação de máquinas e equipamentos & 125,66 & 112,37 & 84,44 \\
\hline 4 - Seguro de máquinas & 5,10 & 4,84 & 3,43 \\
\hline 5 - Correção do solo & 34,90 & 38,43 & 35,32 \\
\hline 6 - Impostos & 7,92 & 8,44 & 9,17 \\
\hline Sub-total (A) & $189,01 a$ & $174,09 a$ & $136,59 a$ \\
\hline 7 - Remuneração do capital máq. e equip. & 55,58 & 48,21 & 36,50 \\
\hline 8 - Remuneração do capital benfeitorias & 13,22 & 8,58 & 3,63 \\
\hline 9 - Remuneração do empresário & 180,75 & 201,97 & 174,63 \\
\hline 10 - Remuneração da terra & 158,33 & 168,75 & 183,33 \\
\hline Sub-total $(B)$ & $407,89 a$ & $427,51 a$ & $398,10 a$ \\
\hline Custo operacional total (COT) & 2.448,43ab & $2.698,69 a$ & $2.319,50 b$ \\
\hline Custo total $(\mathrm{CT})$ & $2.856,32 \mathrm{a}$ & $3.126,20 \mathrm{a}$ & $2.717,60 \mathrm{a}$ \\
\hline
\end{tabular}

Notas: 1) Médias seguidas de mesma letra na linha não diferem entre si pelo teste SNK a 10\% de significância. 2) Valores nominais de maio de 2013.

Tabela 2: Indicadores técnicos e econômicos da produção de milho em área de plantio direto com adubação mineral e orgânica (PDO), com apenas adubação mineral (PDM) e com Integração Lavoura-Pecuária (PDI)

\begin{tabular}{|c|c|c|c|c|}
\hline \multirow[b]{2}{*}{ Especificação } & \multirow[b]{2}{*}{ Unidade } & \multicolumn{3}{|c|}{ Sistema plantio direto } \\
\hline & & $\begin{array}{c}\text { PDO } \\
\text { (com adubação } \\
\text { mineral e } \\
\text { orgânica) }\end{array}$ & $\begin{array}{c}\text { PDM } \\
\text { (com apenas } \\
\text { adubação } \\
\text { mineral) }\end{array}$ & $\begin{array}{c}\text { PDI } \\
\text { (Integração } \\
\text { Lavoura-Pecuária) }\end{array}$ \\
\hline Receita b(RB) & $\mathrm{R} \$ / \mathrm{ha}$ & $4.969,52 \mathrm{a}$ & $4.580,84 \mathrm{a}$ & $4.246,73 a$ \\
\hline Margem bruta (MB) & $\mathrm{R} \$ / \mathrm{ha}$ & $2.710,10 \mathrm{a}$ & $2.056,25 \mathrm{a}$ & $2.063,82 \mathrm{a}$ \\
\hline Receita líquida (RL) & $\mathrm{R} \$ / \mathrm{ha}$ & $2.521,09 \mathrm{a}$ & $1.882,15 \mathrm{a}$ & $1.927,23 \mathrm{a}$ \\
\hline Lucro puro (LUCRO) & $\mathrm{R} \$ / \mathrm{ha}$ & $2.113,20 \mathrm{a}$ & $1.454,64 \mathrm{a}$ & $1.529,13 \mathrm{a}$ \\
\hline Ponto de nivelamento (PN) & sc/ha & $99,45 \mathrm{a}$ & $108,85 \mathrm{a}$ & $94,62 \mathrm{a}$ \\
\hline Taxa de remuneração da terra (TRT) & $\%$ & $9,34 \mathrm{a}$ & $4,90 \mathrm{a}$ & $4,95 \mathrm{a}$ \\
\hline Produtividade total dos fatores (PTF) & un & $1,74 \mathrm{a}$ & $1,47 \mathrm{a}$ & $1,58 \mathrm{a}$ \\
\hline Produtividade por área (PRODA) & $\mathrm{kg} / \mathrm{ha}$ & $10.382,00 \mathrm{a}$ & $9.570,00 \mathrm{a}$ & $8.872,00 \mathrm{a}$ \\
\hline Produtividade da mecanização (PRODMEC) & $\mathrm{kg} / \mathrm{ha} /$ hora máq. & $2.737,56 a$ & $3.166,01 \mathrm{a}$ & $3.614,62 \mathrm{a}$ \\
\hline \multirow{3}{*}{$\begin{array}{l}\text { Produtividade da fertilização } \\
\text { (PROD-N, PROD-P e PROD-K) }\end{array}$} & $\mathrm{kg} / \mathrm{ha} / \mathrm{kg} \mathrm{N}$ & $85,45 \mathrm{a}$ & $43,18 b$ & $47,67 b$ \\
\hline & $\mathrm{kg} / \mathrm{ha} / \mathrm{kg} \mathrm{P}_{2} \mathrm{O}_{5}$ & $165,26 a$ & $82,65 b$ & $81,55 \mathrm{~b}$ \\
\hline & $\mathrm{kg} / \mathrm{ha} / \mathrm{kg} \mathrm{K}{ }_{2} \mathrm{O}$ & $286,88 \mathrm{a}$ & $162,53 \mathrm{a}$ & $232,53 \mathrm{a}$ \\
\hline
\end{tabular}

Notas: Médias seguidas de mesma letra na linha não diferem entre si pelo teste SNK a 10\% de significância. 2) Valores nominais de maio de 2013 
CT e CF-B), o que requer maior nível de produção para cobrir os custos (maior ponto de nivelamento - PN). Já o PDI apresentou associação com maior produtividade da mecanização (PRODMEC). Estes resultados indicam que o plantio direto sem uso de adubação orgânica (PDM) apresenta maior custo de produção, principalmente, por necessidade de se usar mais insumos, fundamentalmente por causa do maior uso de adubação mineral. De acordo com os dados coletados com os produtores, o uso de adubação orgânica reduz a necessidade de adubação mineral de base e de cobertura e proporciona aumento da produtividade da cultura de milho e, consequentemente, melhores resultados econômicos.

Quanto às variáveis explicativas referentes ao solo, o PDO tem maiores valores de $\mathrm{pH}, \mathrm{Pe} \mathrm{K}$ (Figura 2). No que tange ao pH, Scherer et al. (2012), em trabalho avaliando

Tabela 3: Valores médios dos atributos químicos e físicos do solo de áreas de plantio direto com adubação mineral e orgânica (PDO), com apenas adubação mineral (PDM) e com Integração Lavoura-Pecuária (PDI)

\begin{tabular}{|c|c|c|c|c|}
\hline \multirow[b]{2}{*}{ Variáveis explicativas } & \multirow[b]{2}{*}{ Unidade } & \multicolumn{3}{|c|}{ Sistema plantio direto } \\
\hline & & $\begin{array}{c}\text { PDO } \\
\text { (com adubação e } \\
\text { orgânica) }\end{array}$ & $\begin{array}{c}\text { PDM } \\
\text { (com apenas } \\
\text { adubação mineral) }\end{array}$ & $\begin{array}{c}\text { PDI } \\
\text { (Integração Lavoura- } \\
\text { Pecuária) }\end{array}$ \\
\hline pH - pH em água & un & $5,5 \mathrm{a}$ & $5,4 \mathrm{a}$ & $5,2 \mathrm{a}$ \\
\hline P - Fósforo & $\mathrm{mg} / \mathrm{dm}^{3}$ & $31,1 \mathrm{a}$ & $11,9 b$ & $7,1 \mathrm{~b}$ \\
\hline K - Potássio & $\mathrm{mg} / \mathrm{dm}^{3}$ & $338,9 \mathrm{a}$ & $261,5 \mathrm{a}$ & $279,9 \mathrm{a}$ \\
\hline MO - Matéria orgânica & $\%$ & $3,9 \mathrm{a}$ & $4,4 \mathrm{a}$ & $4,6 \mathrm{a}$ \\
\hline Al - Alumínio & $\mathrm{cmol} / \mathrm{dm}^{3}$ & $0,3 \mathrm{a}$ & $0,6 \mathrm{a}$ & $0,9 \mathrm{a}$ \\
\hline Ca - Cálcio & $\mathrm{cmol} / \mathrm{dm}^{3}$ & $6,0 \mathrm{a}$ & $5,9 \mathrm{a}$ & $5,2 \mathrm{a}$ \\
\hline Mg - Magnésio & $\mathrm{cmol} / \mathrm{dm}^{3}$ & $2,2 \mathrm{a}$ & $2,7 \mathrm{a}$ & $2,2 \mathrm{a}$ \\
\hline DS - Densidade do solo & $\mathrm{g} / \mathrm{cm}^{3}$ & $1,19 \mathrm{a}$ & $1,21 \mathrm{a}$ & $1,16 \mathrm{a}$ \\
\hline RP - Resistência à penetração & $\mathrm{kgf} / \mathrm{cm}^{2}$ & $2,76 a$ & $2,52 \mathrm{a}$ & $2,29 \mathrm{a}$ \\
\hline PT - Porosidade total & $\mathrm{cm}^{3} / \mathrm{cm}^{3}$ & $0,54 \mathrm{a}$ & $0,56 \mathrm{a}$ & $0,55 \mathrm{a}$ \\
\hline MacroP - Macroporosidade & $\mathrm{cm}^{3} / \mathrm{cm}^{3}$ & $0,13 \mathrm{a}$ & $0,12 \mathrm{a}$ & $0,14 \mathrm{a}$ \\
\hline MicroP - Microporosidade & $\mathrm{cm}^{3} / \mathrm{cm}^{3}$ & $0,38 \mathrm{a}$ & $0,40 \mathrm{a}$ & $0,37 \mathrm{a}$ \\
\hline DMG - Diâmetro médio geométrico & $\mathrm{mm}$ & $1,95 \mathrm{a}$ & $2,07 \mathrm{a}$ & $1,86 \mathrm{a}$ \\
\hline
\end{tabular}

Nota: Médias seguidas de mesma letra na linha não diferem entre si pelo teste SNK a 10\% de significância.
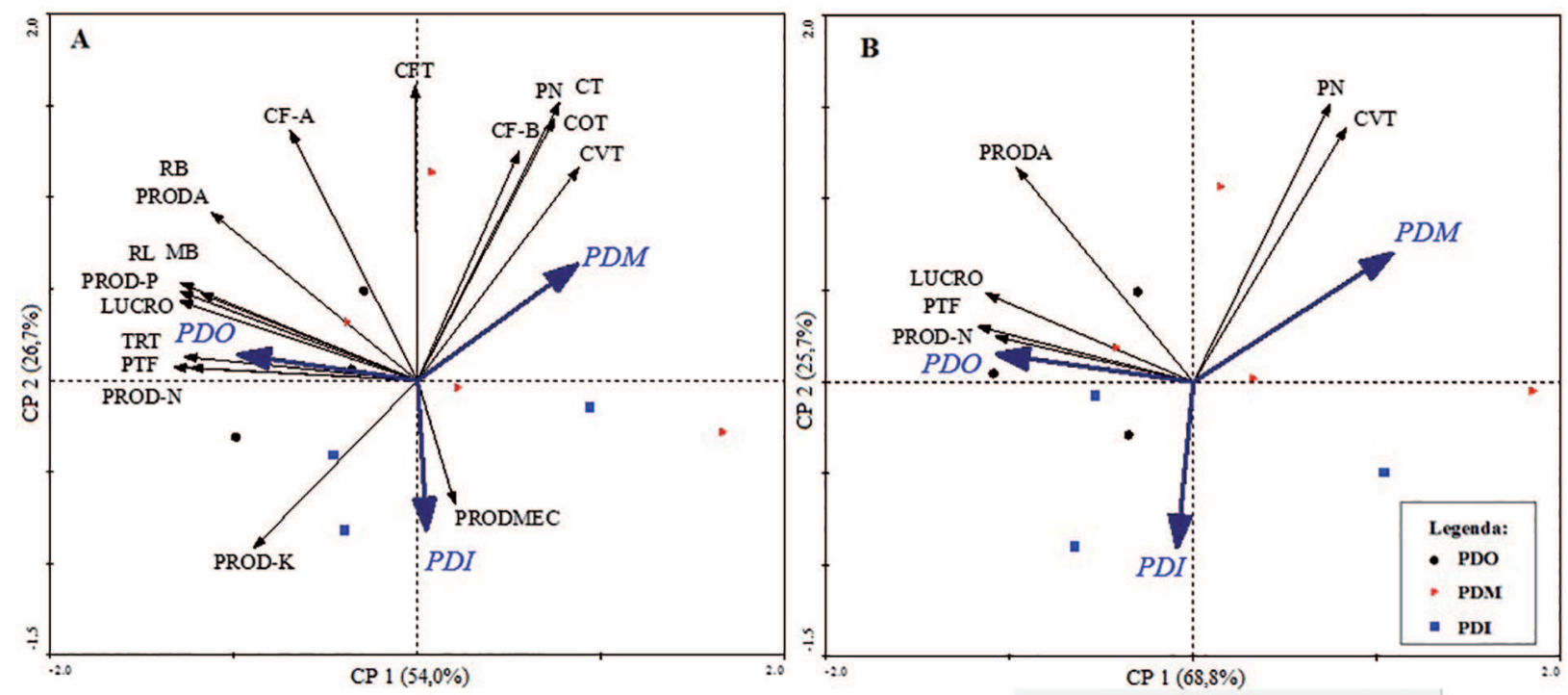

Figura 1: Relação entre a componente principal 1 ( $\mathrm{CP} 1$ em $\mathrm{A}=54,0 \%$ e em $\mathrm{B}=68,8 \%$ ) e $2(\mathrm{CP} 2$ em $\mathrm{A}=26,7 \%$ e em $\mathrm{B}=25,7 \%)$, discriminando-se os diferentes manejos do solo, com uso de apenas adubação mineral (PDM), adubação mineral e orgânica (PDO) e integração lavoura-pecuária (PDI), variáveis respostas (A) e variáveis respostas selecionadas (B): Custo Variável Total (CVT), Custo Fixo Total (CFT), Custo Operacional Total (COT), Custo Total (CT), Receita Bruta (RB), Margem Bruta (MB), Receita Líquida (RL), Lucro Puro (LUCRO), Ponto de Nivelamento (PN), Taxa de Remuneração da Terra (TRT), Produtividade Total dos Fatores (PTF), Produtividade por área (PRODA), Produtividade da mecanização (PRODMEC), Produtividade da fertilização - nitrogênio (PROD-N), Fósforo (PROD-P) e potássio (PROD-K). 


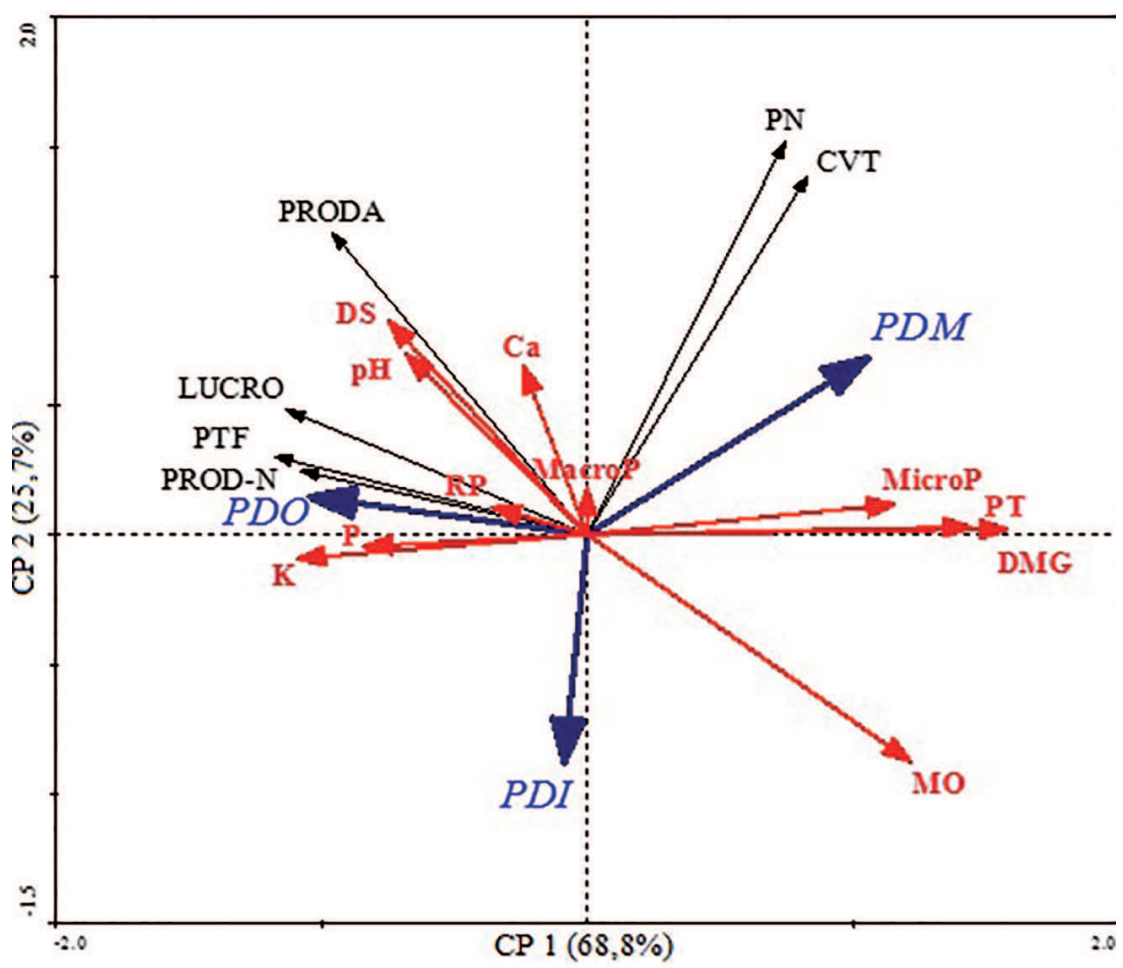

Figura 2: Relação entre a componente principal 1 (CP1=68,8\%) e 2 (CP2=25,7\%), discriminando-se os diferentes manejos do solo, com uso de apenas adubação mineral (PDM), adubação mineral e orgânica (PDO) e integração lavoura-pecuária (PDI) e suas variáveis respostas (CVT: Custo Variável Total; LUCRO: Lucro Puro; PN: Ponto de Nivelamento; PTF: Produtividade Total dos Fatores; PRODA: Produtividade por área; PROD-N: Produtividade da fertilização nitrogenada) e explicativas (pH: PH em água; Ca: cálcio; P: fósforo; K: potássio; MO: matéria orgânica; DS: Densidade do solo; RP: resistência à penetração; PT: volume total de poros; MacroP: volume de macroporos; MicroP: volume de microporos; DMG: diâmetro médio geométrico dos agregados secos ao ar).

atributos de solo influenciados pelas adubações orgânica e nitrogenada, encontrou relatos de efeitos da adubação orgânica positivos, negativos e, mesmo, ausentes. De qualquer modo, após 13 anos de cultivo, a associação da aplicação de dejetos líquidos e da calagem recomendada proporcionou valores de $\mathrm{pH}$ acima dos encontrados nas demais modalidades. Isso pode estar associado à presença de cal na cama de aviário, que, quando reage no solo, resulta na elevação do $\mathrm{pH}$, conforme também observado por Veiga et al. (2006) em estudo com aplicação de diferentes fontes de nutrientes em um Nitossolo Vermelho. Em relação aos valores de P disponível e K trocável, as maiores concentrações em PDO resultam do efeito acumulativo que o uso contínuo de fertilizantes orgânicos, complementado por fertilizantes minerais, proporciona ao solo. Neste sentido, Scherer et al. (2013), avaliando áreas com aplicação intensiva de dejetos líquidos de suínos, encontraram, na camada $0-10 \mathrm{~cm}$, teores de $\mathrm{P}$ e de $\mathrm{K}$ altos ou muito altos, em $97 \%$ das áreas avaliadas, considerando-se as faixas estabelecidas pela Comissão de Fertilidade do Solo para os Estados do RS e SC, da Sociedade Brasileira de Ciência do Solo (SBCS, 2016).

No que se refere aos atributos físicos do solo, DS e RP situam-se no quadrante do PDO, sendo o segundo com magnitude muito reduzida. A maior DS no PDO pode ser devida ao uso de caminhão ou trator com distribuidor para aplicação do adubo orgânico, entre outras operações com máquinas e equipamentos realizadas na área, o que pode provocar compactação do solo, principalmente na camada superficial, em função de a pressão aplicada pelo rodado ser maior do que a resistência do solo (Hakansson \& Voorhees, 1998). Os atributos PT e MicroP estão associados ao PDM e ao PDI, sendo mais relacionados com o primeiro, mas com magnitude similar entre eles. Como é de se esperar, os maiores valores desses dois atributos estão associados com a menor densidade do solo encontrada nessas modalidades.

O PDM se associa melhor com MO, DMG e porosidade total (PT), esta, fundamentalmente por causa de maior MicroP. Esses valores estão relacionados com a menor ação antrópica no sistema e se correlacionam entre si, ou seja, as três variáveis são dependentes umas das outras. Como no PD, em que se esperava uma menor oxidação da MO, que normalmente está associada à ocorrência de maior DMG e porosidade total, o que se reflete em um maior valor desses atributos, embora não tenha sido detectada diferença estatística significativa. O sistema PDI foi o menos associado com os diversos atributos do solo; 
contudo, apresentou a maior associação com o atributo MO. De acordo com as entrevistas de campo, no tratamento PDI há redução da produtividade da cultura de verão, entre 5 e $10 \%$. Contudo, os ganhos com a produção de bovinos são compensatórios, de modo a proporcionar um saldo financeiro anual positivo.

Considerando-se os resultados da associação de diversos indicadores com as diferentes variações de manejo nos SPD estudados, propiciados pela análise multivariada usada neste estudo, pode-se considerar que essa ferramenta estatística é adequada para demonstrar as relações existentes entre os indicadores técnicos e econômicos e as variáveis respostas. Essa análise possibilita uma visão mais global do efeito dos tratamentos, recomendando-se seu uso em estudos desta natureza.

\section{CONCLUSÕES}

As diferentes variações de manejo em SPD estudadas apresentaram efeitos significativos sobre o custo operacional total, a resposta à fertilização com $\mathrm{N}$ e $\mathrm{P}$ e os níveis de $\mathrm{P}$ disponíveis no solo. O menor custo de produção de milho foi observado no manejo com integração lavoura-pecuária, mas os melhores resultados econômicos ocorreram no manejo lavoura com adubação orgânica (PDO). Esse sistema reduziu os custos (fundamentalmente graças ao menor uso de adubação mineral) e proporcionou maiores índices de produtividade e de eficiências técnica e econômica na produção, contribuindo para a maior sustentabilidade do sistema de produção. Adicionalmente, a análise multivariada mostrou-se uma ferramenta mais apropriada para interpretar e explicar os resultados obtidos nos diferentes manejos estudados.

\section{REFERÊNCIAS}

Alvim MISA, Valle SML, Lima JE \& Silva OM (2004) Análise da competitividade da produção de soja nos sistemas de plantio direto e plantio convencional na região do cerrado brasileiro. Revista de Economia e Sociologia Rural, 42:223-243.

Baretta D, Santos JCP, Mafra AL, Wildner L do P \& Miquelluti DJ (2003) Fauna edáfica avaliada por armadilhas e catação manual afetada pelo manejo do solo na região oeste catarinense. Revista de Ciências Agroveterinárias, 2:97-106.

Bartz MLC, Pasini A \& Brown GG (2013) Earthworms as soil quality indicators in Brazilian No-tillage systems. Applied Soil Ecology, 69:39-48.

Bartz MLC, Brown GG, Rosa MG, Klauberg-Filho O, James SW, Decaëns T \& Baretta D (2014) Earthworm richness in land-use systems in Santa Catarina, Brazil. Applied Soil Ecology, 83:59-70.

Braak CJF \& Smilaeur P (1998) Reference manual and user's guide to CANOCO for windows: Software for Canonical Community Ordination (version 4). Ithaca, Microcomputer Power. 500 p.

Brown GG, Bennito NP, Pasini A, Sautter KD, Guimarães MF \& Torres E (2003) No-tillage greatly increases earthworm populations in Paraná state, Brazil. Pedobiologia, 47:05-06.
Conab (2010) Custos de produção agrícola: a metodologia da Conab. Disponível em: <http://www.conab.gov.br/conabweb/download/ safra/custos.pdf>. Acessado em: 17 de junho de 2013.

Conterato MA, Fernandes LL, Libardoni PJ, Gomes MC \& Stein A de Q (2012) Evolução da agropecuária brasileira: eficiência e produtividade na vanguarda de um modelo de desenvolvimento rural. In: $50^{\circ}$ Congresso da Sociedade Brasileira de Economia e Sociologia Rural, Vitória. Anais, SOBER. p. 1-19.

Cruz SCS, Pereira FRS, Bicudo SJ, Santos JR, Alburquerque AW \& Machado CG (2009) Consórcio de milho e Brachiaria decumbens em diferentes preparos de solo. Acta Scientiarum. Agronomy, 31:633-639.

Cusumano V, Rezende JLP \& Kirst PH (1975) Uma análise de produtividade agrícola: efeito do capital na renda agrícola do município de Santa Maria, (RS). Revista do Centro de Ciências Rurais, 5:209-218.

Derpsch R, Roth CH, Sidiras N \& Köpke U (1991) Controle da erosão no Paraná, Brasil: sistemas de cobertura do solo, plantio direto e preparo conservacionista do solo. Eschborn, Deutsche Gesellschaft für Technische Zusammenarbeit (GTZ). 272p.

Epagri (2014a) Síntese Anual da Agricultura de Santa Catarina 2012-2013. Disponível em: <http://docweb.epagri.sc.gov.br/ website_cepa/publicacoes/sintese_2013.pdf>. Acessado em: 03 de setembro de 2014.

Epagri (2014b) Custo de produção. Disponível em: <http:// www.epagri.sc.gov.br/?page_id=2696>. Acessado em: 03 de setembro de 2014.

Gomes JA, Scapim CA, Branccini A de Le, Vidigal Filho PS, Sagrilo E \& Mora F (2005) Adubações orgânica e mineral, produtividade do milho e características físicas e químicas de um Argissolo Vermelho Amarelo. Acta Scientiarum. Biological Sciences, 27:521-529.

Hakansson I \& Voorhees WB (1998) Soil compaction. In: Lal R, Bum WH, Valentine C \& Stewart BA (Ed.) Methods for assessment of soil degradation. Boca Raton, CRC Press. p.167179.

Hoffmann R, Engler JJ de, Serrano O, Thame AC de M \& Neves EM (1981) Administração da empresa agrícola. $3^{a}$ ed. São Paulo, Pioneira. 325p.

Ibge (2014) Banco de dados agregados: produção agrícola municipal. Disponível em: <http://www.sidra.ibge.gov.br/bda/pesquisas/ pam/default.asp?o=18\&i=P $>$. Acessado em: 03 de setembro de 2014.

Kageyama A (2003) Produtividade e renda na agricultura familiar: efeitos do PRONAF-crédito. Agricultura em São Paulo, 50:01-13.

Kaneko FH, Arf O, Gitti DC, Tarsitano MAA, Rapasi RMA \& Vilala RG (2010) Custos e rentabilidade do milho em função do manejo do solo e da adubação nitrogenada. Pesquisa Agropecuária Tropical, 40:102-109.

Leps J \& Smilauer P (1999) Multivariate analysis of ecological data using CANOCO. Ceské Budejovice, University of South Bohemia. 110p.

Mapa (2014) Culturas: milho. Disponível em: <http:// www.agricultura.gov.br/vegetal/culturas/milho>. Acessado em: 03 de setembro de 2014.

Pereira J de M, Baretta D \& Cardoso EJBN (2013) Relação entre atividade microbiana a atributos físicos e químicos do solo em florestas de Araucaria angustifolia nativa e replantada no Estado de São Paulo. Revista Brasileira de Ciência do Solo, 37:01-08.

Pieri C, Evers G, Landers J, O’Connell P \& Terry E (2002) No-till farming for sustainable rural development. Washington, Agriculture and Rural Development Working Paper. 77p. 
Possamai JM, Souza CM \& Galvão JCC (2001) Sistemas de preparo do solo para o cultivo do milho safrinha. Bragantia, 60:7982 .

Santos HP, Ambrosi I, Lhamby JCB \& Carmo C (2004) Lucratividade e risco de sistemas de manejo de solo e de rotação e sucessão de culturas. Ciência Rural, 34:97-103.

Scherer EE, Baldissera IT \& Miranda M (2012) Produção e qualidade e forragem e atributos do solo influenciados por adubação orgânica e nitrogenada. Florianópolis, Epagri. 50p. (Boletim técnico, 157).

Scherer EE, Baldissera IT \& Spagnollo E (2013) Qualidade do solo e da água surgente em áreas com uso intensivo e continuado de esterco de suínos como fertilizante. Florianópolis, Epagri. 38p. (Boletim Técnico, 160).

SEAB (2013) Custos de produção. Disponível em: <http:// www.agricultura.pr.gov.br/>. Acessado em: 03 de setembro de 2014.

Silva JG, Kageyama AA, Romão DA, Wagner Neto JA \& Pinto LCG (1983) Tecnologia e campesinato: o caso brasileiro. Revista de Economia Política, 3:21-56.

Silveira PM, Silva OF, Stone LF \& Silva JG (2001) Efeitos do preparo do solo, plantio direto e de rotações de culturas sobre o rendimento e a economicidade do feijoeiro irrigado. Pesquisa Agropecuária Brasileira, 36:257-63
SBCS (2016) Manual de adubação e de calagem para os estados do Rio Grande do Sul e de Santa Catarina. 11 ${ }^{\mathrm{a}}$ ed. Porto Alegre, Sociedade Brasileira de Ciência do Solo/Núcleo Regional Sul. $376 \mathrm{p}$.

Tedesco MJ, Gianello C, Bissani CA, Bohnen H \& Volkweiss SJ (1995) Análises de solo, plantas e outros materiais. Porto Alegre, Universidade Federal do Rio Grande do Sul. 174p. (Boletim técnico, 5).

Veiga M, Reinert DJ \& Pandolfo CM (2006) Efeito de sistemas de preparo e de fontes de nutrientes sobre a fertilidade do solo e o crescimento e produção de milho. Agropecuária Catarinense, 19:69-73.

Veiga M (2011) Metodologia para coleta de amostras e análises físicas do solo. Florianópolis, Epagri. 52p. (Boletim Técnico, 156).

Veiga M, Zardo VF \& Pandolfo CM (2014) Atributos do solo em função da oferta de pastagem anual de inverno para terminação de bovinos, em sistema integração lavoura-pecuária. In: 10 Reunião Sul-Brasileira de Ciência do Solo, Pelotas. Anais, NRSSBCS. p.1-4 